\title{
A new index for distinguishing hypereosinophilic syndrome and antineutrophil cytoplasmic antibody-negative eosinophilic granulomatosis with polyangiitis
}

\author{
Sung Soo Ahn, ${ }^{1}$ Juyoung Yoo, ${ }^{1}$ Yong-Beom Park, ${ }^{1,2}$ Jung-Won Park, ${ }^{3,4}$ Jae-Hyun Lee, ${ }^{3,4 *}$ Sang-Won Lee, ${ }^{1,2 *}$
}

\begin{abstract}
Background: It is difficult to differentiate between hypereosinophilic syndrome (HES) and antineutrophil cytoplasmic antibody (ANCA)-negative eosinophilic granulomatosis with polyangiitis (EGPA).

Objective: We compared laboratory data at diagnosis between Korean patients with HES and ANCA-negative EGPA and investigated independent laboratory predictors suggesting HES.
\end{abstract}

Methods: We reviewed the medical records of 41 HES patients and 16 ANCA-negative EGPA patients. The cut-offs were extrapolated by the receiver operator characteristic (ROC) curve. The odds ratio (OR) and relative risk (RR) were assessed using the multivariable logistic regression analysis and the chi-square test, respectively. We developed a new equation by assigning a weight to each variable according to the slopes (B) and expressed a decimal as the nearest integer.

Results: HES patients had a higher median WBC and eosinophil counts than ANCA-negative EGPA patients. The cutoffs of WBC and eosinophil counts for HES were set at $9,900.0 / \mathrm{mm}^{3}$ and $2,400.0 / \mathrm{mm}^{3}$. In the multivariable analysis, WBC count $\geq 9,900.0 / \mathrm{mm}^{3}$ (B 1.763) and eosinophil count $\geq 2,400.0 / \mathrm{mm}^{3}$ (B 1.515) were significantly associated with HES. An equation was as follows: HES-suggesting laboratory index (HSLI) $=2 \times\left(\right.$ WBC count $\geq 9,900.0 / \mathrm{mm}^{3}(1=\mathrm{No}$ or 2 = Yes $))+1.5 \times\left(\right.$ eosinophil count $\geq 2,400.0 / \mathrm{mm}^{3}(1=$ No or $2=$ Yes $\left.)\right)$. The cut-off of HSLI for HES was 4.25 . Patients with HSLI $\geq 4.25$ exhibited a significantly high RR (51.429) for HES, compared to those without.

Conclusions: In conclusion, the cut-off of HSLI derived from WBC and eosinophil counts could be an independent predictor of HES in patients suspected of both HES and ANCA-negative EGPA.

Key words: hypereosinophilic syndrome, eosinophilic granulomatosis with polyangiitis, predictor, differentiation, index

\footnotetext{
From:

Division of Rheumatology, Department of Internal Medicine, Yonsei University College of Medicine, Seoul, Republic of Korea Institute for Immunology and Immunological Diseases, Yonsei University College of Medicine, Seoul, Republic of Korea Division of Allergy, Department of Internal Medicine, Yonsei University College of Medicine, Seoul, Republic of Korea ${ }^{4}$ Institute of Allergy, Yonsei University College of Medicine, Seoul, Republic of Korea

* Contributed equally to this work.
}

\section{Introduction}

Hypereosinophilic syndrome (HES) is a group of conditions including marked peripheral hypereosinophilia and end-organ eosinophil infiltration, leading to organ damages by uncertain aetiologies. ${ }^{1}$ The first diagnostic criteria for HES were established by Chusid et al. in 1975 and were modified

\author{
Corresponding author: \\ 1. Sang-Won Lee \\ Division of Rheumatology, Department of Internal Medicine, \\ Institute for Immunology and Immunological Diseases, \\ Yonsei University College of Medicine, \\ 50-1 Yonsei-ro, Seodaemun-gu, Seoul, 03722, Republic of Korea \\ E-mail: sangwonlee@yuhs.ac \\ 2. Jae-Hyun Lee \\ Division of Allergy, Department of Internal Medicine, \\ Institute of Allergy, Yonsei University College of Medicine \\ 50-1 Yonsei-ro, Seodaemun-gu, Seoul, 03722, Republic of Korea \\ E-mail: JHLEEMD@yuhs.ac
}

by Klion et al. in $20066^{2,3}$ The refined definition of HES, which was proposed in 2010 and has currently been used, includes i) blood eosinophils $>1500 / \mathrm{mm}^{3}$ on at least 2 occasions or evidence of prominent tissue eosinophilia associated with symptoms regardless of peripheral blood eosinophilia and 
ii) exclusion of secondary cause of eosinophilia. According to this definition, absolute hypereosinophilia (blood eosinophils $>1500 / \mathrm{mm}^{3}$ ) is not obligatorily required in a case of endorgan dysfunction. ${ }^{4}$

The classification of eosinophilic granulomatosis with polyangiitis (EGPA), which is identically called Churg-Strauss syndrome (CSS), was first proposed by Churg and Strauss in 1951. They defined CSS as vasculitis of small to medium vessels and necrotizing inflammation with extravascular granulomatosis in patients with asthma or tissue eosinophilia. ${ }^{5}$ EGPA is one of antineutrophil cytoplasmic antibody (ANCA)-associated vasculitis (AAV) along with microscopic polyangiitis (MPA) and granulomatosis with polyangiitis (GPA). According to the 2012 Chapel Hill Consensus Conferences Nomenclature of Vasculitis (the 2012 CHCC definitions), on the basis of small-vessel necrotising vasculitis, EGPA is characterised by three typical allergic components including asthma, peripheral eosinophilia and eosinophil-rich granuloma of the respiratory tracts. ${ }^{6}$ The American College of Rheumatology 1990 criteria for classification (the 1990 ACR criteria) for Churg-Strauss syndrome, which have currently been used, include 6 items as the following: i) asthma, ii) paranasal sinus abnormality, iii) peripheral blood eosinophilia (> 10\%), iv) unfixed pulmonary infiltration, v) mononeuropathy or polyneuropathy and vi) extravascular eosinophils on histology. EGPA can be diagnosed when four or more of the above 6 items are satisfied. $^{7}$

In real clinical settings, it is difficult to differentiate between HES and EGPA due to common allergic components such as peripheral blood eosinophilia and eosinophil infiltration on histology and clinical similarities such as paranasal sinusitis and eosinophilic pneumonia. ${ }^{4,7}$ Asthma could be considered a favourable clue for EGPA, as all potential causes of hypereosinophilia should be excluded in HES. ${ }^{4}$ However, asthma may occur as a consequence of HES. ${ }^{9}$ In addition, ANCA positivity can be definitive evidence to differentiate EGPA from HES. However, in EGPA, ANCA is detected only in 30 to $50 \%$ percent of patients, which is half the rate of MPA and GPA patients. ${ }^{10,11}$ Thus, ANCA positivity is useless for distinguishing between HES and ANCAnegative EGPA.

On the other hands, HES is currently divided into 6 categories such as myeloproliferative HES, lymphocytic HES, undefined HES, overlap HES, associated HES and familial HES. The category of associated HES, which is composed of significant peripheral eosinophilia under the conditions known to provoke eosinophilia, includes EGPA., ${ }^{40,12}$ Therefore, it is questioned whether distinguishing HES from EGPA has a clinical implication. Nevertheless, it is important to distinguish the two disease, for therapeutic approaches are different. Therapeutic regimens for HES are mainly determined based on the category, whereas those for EGPA are primarily decided according to five-factor score (FFS) suggested by the French Vasculitis Study Group..$^{10,12-14}$

So far, there have been several efforts to identify serum biomarkers for distinguishing between HES and ANCA-negative EGPA but there is no biomarker to clearly divide the two diseases yet. ${ }^{15,16}$ In addition, although ANCA-negative EGPA is not common, since the differential diagnosis between two diseases are challenging, a development of novel biomarker is necessary. Furthermore, there has been no objective indicator using laboratory data to help to differentiate the two disease to date. Hence, in this study, we compared laboratory data at diagnosis between Korean patients with established HES and ANCA-negative EGPA and investigated independent laboratory predictors suggesting HES.

\section{Methods \\ Patients}

We retrospectively reviewed the medical records of Korean immunosuppressive drug-naïve patients with either HES and ANCA-negative EGPA (41 HES patients and 16 ANCAnegative EGPA patients). All patients were first classified as HES or EGPA at the Department of Internal Medicine, Yonsei University College of Medicine, Severance Hospital, from November 2005 to November 2018. We reclassified HES patients based on 2010 Refining the definition of HES and EGPA patients based on the 1990 ACR criteria, the 2007 European Medicine Agency algorithm and the 2012 CHCC definitions. ${ }^{6,717}$ They had well-documented medical records with which to assess clinical manifestations and organ damages at diagnosis. We excluded patients who had concurrent medical conditions to affect clinical and laboratory data at diagnosis, such as serious infections, particularly parasite infections, solid malignancies, haematological proliferative diseases and other types of inflammatory disease. We also excluded patients who received immunosuppressive drugs for HES or EGPA prior to diagnosis, which were verified by the Korean Drug Utilisation Review (DUR) system. This study was approved by the Institutional Review Board (IRB) of Severance Hospital (4-2017-0673), who waived the need for patient written informed consent, as this was a retrospective study.

\section{Clinical manifestations and laboratory results}

We evaluated how many items of the 1990 ACR criteria for CSS were met in HES and ANCA-negative EGPA patients. Organ specific clinical manifestation at diagnosis were reviewed. Laboratory results at diagnosis were obtained by the automatic Clinical Data Repository system in our institute. They include ANCA positivity, white blood cell (WBC) count, eosinophil percentage and count, haemoglobin, platelet count, blood urea nitrogen, creatinine, alkaline phosphatase, erythrocyte sedimentation rate (ESR) and C-reactive protein (CRP).

\section{Statistical analyses}

All statistical analyses were conducted using SPSS software (version 23 for windows; IBM Corp., Armonk, NY, USA). Owing to the small sample size, continuous variables were expressed as a median (interquartile range, IQR) and categorical variables were expressed as number and the percentage. Significant differences in categorical variables between the two groups were compared using the chi square and Fisher's exact tests. Significant differences in continuous variables between the two groups were compared using the Mann-Whitney test. The optimal cut-offs were extrapolated by calculating the receiver operator characteristic (ROC) curve 
and selecting the maximised sum of sensitivity and specificity. We developed a new equation by assigning a weight to each variable according to the slopes (B) in the multivariable logistic regression analysis and expressed a decimal as the nearest integer. The odds ratio (OR) was assessed using the multivariable logistic regression analysis of laboratory variables with $p$-values less than 0.05 on the univariable logistic regression analysis. The relative risk (RR) was analysed using contingency tables and the chi-square test. $P$-values less than 0.05 were considered statistically significant.

\section{Results}

\section{Comparison of baseline characteristics}

Comparison of baseline characteristics between patients with HES and ANCA-negative EGPA are described in Table 1. The median age at diagnosis was 50.0 years and 33 patients were men $(57.9 \%)$. There were no differences in age at diagnosis and gender between two groups. Among ANCA-negative EGPA patients, 13 patients met 4 items of the 1990 ACR criteria for EGPA, 1 patient met 5 items and 2 patients met 6 items. Asthma, paranasal sinus abnormality, mononeuropathy or polyneuropathy, and extravascular eosinophils on histology were observed in ANCA-negative EGPA patients more frequently than HES patients. Meanwhile, all HES patients exhibited peripheral blood eosinophilia. Among clinical manifestations at diagnosis, ANCA-negative EGPA patients exhibited ear nose throat (ENT), renal, and nervous manifestations more frequently than HES patients. Among laboratory results at diagnosis, the median WBC and eosinophil counts in HES patients were significantly higher than those in ANCAnegative EGPA patients $\left(13,850.0 / \mathrm{mm}^{3}\right.$ vs. $7,450.0 / \mathrm{mm}^{3}, P=$ 0.033 and $5,290.0 / \mathrm{mm}^{3}$ vs. $1,910.0 / \mathrm{mm}^{3}, P=0.039$, respectively).

Table 1. Comparison of clinical and laboratory data between patients with HES and ANCA-negative EGPA

\begin{tabular}{|c|c|c|c|}
\hline Variables & $\begin{array}{l}\text { Patients with } \\
\text { HES } \\
(\mathbf{N}=41)\end{array}$ & $\begin{array}{l}\text { Patients with } \\
\text { ANCA-negative EGPA } \\
\qquad(\mathbf{N}=16)\end{array}$ & $P$-value \\
\hline \multicolumn{4}{|l|}{ Demographic data } \\
\hline Age at diagnosis (year old) & $52.6(34.0)$ & $50.5(20.8)$ & 0.564 \\
\hline Male gender (N (\%)) & $27(65.9)$ & $6(37.5)$ & 0.084 \\
\hline \multicolumn{4}{|c|}{ The 1990 ACR classification criteria for EGPA (N (\%)) at diagnosis } \\
\hline Asthma & $10(24.4)$ & $15(93.8)$ & $<0.001$ \\
\hline Peripheral blood Eosinophilia (>10\%) & $41(100)$ & $16(100)$ & $\mathrm{N} / \mathrm{A}$ \\
\hline Paranasal sinus abnormality & $13(31.7)$ & $15(93.8)$ & $<0.001$ \\
\hline Unfixed pulmonary infiltration & $8(19.5)$ & $5(31.3)$ & 0.343 \\
\hline Mononeuropathy or polyneuropathy & $5(12.2)$ & $9(56.3)$ & 0.001 \\
\hline Extravascular eosinophils on histology & $0(0.0)$ & $10(62.5)$ & $<0.001$ \\
\hline \multicolumn{4}{|l|}{ Clinical manifestations at diagnosis (N (\%)) } \\
\hline Skin & $13(31.7)$ & $5(31.3)$ & 0.716 \\
\hline Eczema & $6(14.6)$ & $0(0.0)$ & \\
\hline Pseudo-urticarial rash & $5(12.2)$ & $2(12.5)$ & \\
\hline Purpura & $1(2.4)$ & $1(6.3)$ & \\
\hline Petechiae & $0(0.0)$ & $1(6.3)$ & \\
\hline Subcutaneous nodule & $0(0.0)$ & $1(6.3)$ & \\
\hline Necrosis & $1(2.4)$ & $0(0.0)$ & \\
\hline Ear, nose and throat & $13(31.7)$ & $15(93.8)$ & $<0.001$ \\
\hline Sinusitis & $13(31.7)$ & $12(75.0)$ & \\
\hline Rhinitis & $1(2.4)$ & $4(25.0)$ & \\
\hline Polyposis & $0(0.0)$ & $1(6.3)$ & \\
\hline Lungs & $8(14.0)$ & $5(31.3)$ & 0.343 \\
\hline Infiltrate & $5(12.2)$ & $4(25.0)$ & \\
\hline Nodule & $2(4.9)$ & $2(12.5)$ & \\
\hline Pleural effusion & $3(7.3)$ & $0(0.0)$ & \\
\hline
\end{tabular}


Table 1. (Continued)

\begin{tabular}{|c|c|c|c|}
\hline Variables & $\begin{array}{c}\text { Patients with } \\
\text { HES } \\
(\mathbf{N}=41)\end{array}$ & $\begin{array}{c}\text { Patients with } \\
\text { ANCA-negative EGPA } \\
\qquad(\mathrm{N}=16)\end{array}$ & $P$-value \\
\hline \multicolumn{4}{|c|}{ Clinical manifestations at diagnosis (N (\%)) (Continued) } \\
\hline Heart & $6(10.5)$ & $4(25.0)$ & 0.355 \\
\hline Cardiomyopathy & $4(9.8)$ & $4(25.0)$ & \\
\hline Pericardial effusion & $3(7.3)$ & $1(6.3)$ & \\
\hline Gastrointestinal tract & $6(10.5)$ & $0(0.0)$ & 0.106 \\
\hline Enteritis & $6(14.6)$ & $0(0.0)$ & \\
\hline Liver & $10(24.4)$ & $1(6.3)$ & 0.119 \\
\hline Focal lesion & $10(24.4)$ & $1(6.3)$ & \\
\hline Kidneys & $0(0)$ & $2(12.5)$ & 0.021 \\
\hline Proteinuria & $0(0.0)$ & $2(12.5)$ & \\
\hline Nerves & $5(12.2)$ & $9(56.3)$ & 0.001 \\
\hline Peripheral neuropathy & $4(9.8)$ & $9(56.3)$ & \\
\hline Mononeuritis multiplex & $1(2.4)$ & $1(6.3)$ & \\
\hline \multicolumn{4}{|l|}{ Laboratory tests at diagnosis } \\
\hline WBC count $\left(/ \mathrm{mm}^{3}\right)$ & $13,850.0(7,450.0)$ & $7,970.0(15,820.0)$ & 0.033 \\
\hline Eosinophil (\%) & $34.0(34.7)$ & $24.3(43.3)$ & 0.263 \\
\hline Eosinophil count $\left(/ \mathrm{mm}^{3}\right)$ & $5,290.0(7,430.0)$ & $1,910.0(10,917.0)$ & 0.039 \\
\hline Haemoglobin (g/dL) & $13.2(2.4)$ & $13.8(0.9)$ & 0.540 \\
\hline Platelet count $\left(\times 1,000 / \mathrm{mm}^{3}\right)$ & $310.0(129.5)$ & $269.0(94.5)$ & 0.248 \\
\hline Blood urea nitrogen $(\mathrm{mg} / \mathrm{dL})$ & $14.2(5.6)$ & $12.3(11.9)$ & 0.479 \\
\hline Creatinine $(\mathrm{mg} / \mathrm{dL})$ & $0.8(0.2)$ & $0.7(0.4)$ & 0.894 \\
\hline Alkaline phosphatase (IU/L) & $72.0(45.5)$ & $77.5(55.0)$ & 0.657 \\
\hline $\operatorname{ESR}(\mathrm{mm} / \mathrm{hr})$ & $20.0(46.0)$ & $33.5(52.8)$ & 0.859 \\
\hline $\mathrm{CRP}(\mathrm{mg} / \mathrm{L})$ & $4.4(15.0)$ & $1.0(30.4)$ & 0.350 \\
\hline
\end{tabular}

Values are expressed as a median (interquartile range, IQR) or number (\%).

HES: hypereosinophilic syndrome; ANCA: antineutrophil cytoplasmic antibody; EGPA: eosinophilic granulomatosis with polyangiitis; ACR: American College of Rheumatology; N/A: not applicable; WBC: white blood cell; ESR: erythrocyte sedimentation rate; CRP: C-reactive protein.

\section{Optimal cut-offs for HES}

Since there were significant differences in WBC and eosinophil counts at diagnosis between HES and ANCA-negative EGPA patients, we calculated the optimal cut-offs of WBC and eosinophil counts for determining HES by selecting the maximised sum of sensitivity and specificity in the ROC curve. The optimal cut-off of WBC count at diagnosis for HES was set at $9,900.0 / \mathrm{mm}^{3}$ (area $0.683,95 \%$ confidence interval (CI) $0.479,0.887$, sensitivity 0.88 and sensitivity 0,69 ) and that of eosinophil count at diagnosis for HES was set at $2,400.0 / \mathrm{mm}^{3}$ (Area $0.683,95 \%$ CI 0.527, 0.700) (Table 2).

\section{HES-suggesting laboratory index (HSLI)}

To find independent predictors for HES, we conducted the multivariable logistic regression analyses with $\mathrm{WBC}$ and eosinophil counts at diagnosis, because none of the other laboratory variables were statistically significant in univariable analysis. In the multivariable analysis, $\mathrm{WBC}$ count $\geq 9,900.0 /$ $\mathrm{mm}^{3}$ (B 1.763) and eosinophil count $\geq 2,400.0 / \mathrm{mm}^{3}$ (B 1.515) were significantly associated with HES (Table 3 ). We derived a novel equation of HSLI using variables with $P$ value $<0.05$ in the multivariable logistic regression analysis. Using these slopes (B) in the multivariable logistic regression analysis, we developed a new equation as follows: HSLI $=2 \times($ WBC count $\geq 9,900.0 / \mathrm{mm}^{3}(1=$ No or $2=$ Yes $\left.)\right)+1.5 \times($ eosinophil count $\geq 2,400.0 / \mathrm{mm}^{3}(1=$ No or $2=$ Yes $)$ ).

\section{Relative risk for HES based on HSLI}

We also obtained the optimal cut-offs of HSLI for HES. The optimal cut-off of HSLI for HES was set at 4.25 (Area $0.772,95 \%$ CI 0.613, 0.931) (Figure 1A). When we divided $\mathrm{AAV}$ patients into the two groups based on the optimal cut-off 
Table 2. Optimal cut-offs of WBC and eosinophil counts at diagnosis for HES

\begin{tabular}{|c|c|c|c|c|c|c|}
\hline Variables & Area & $P$-value & $\begin{array}{c}95 \% \text { confidence } \\
\text { interval }\end{array}$ & Optimal cut-off & Sensitivity & Specificity \\
\hline WBC count $\left(/ \mathrm{mm}^{3}\right)$ & 0.683 & 0.033 & $0.479,0.887$ & $\geq 9,900.0$ & $88 \%$ & $69 \%$ \\
\hline Eosinophil count $\left(/ \mathrm{mm}^{3}\right)$ & 0.614 & 0.044 & $0.527,0.700$ & $\geq 2,400.0$ & $83 \%$ & $63 \%$ \\
\hline
\end{tabular}

WBC: white blood cell; HES: hypereosinophilic syndrome.

Table 3. Logistic regression analyses of laboratory variables at diagnosis for HES

\begin{tabular}{|c|c|c|c|c|c|c|c|c|}
\hline & \multicolumn{4}{|c|}{ Univariable } & \multicolumn{4}{|c|}{ Multivariable } \\
\hline & B & OR & $95 \% \mathrm{CI}$ & $P$ value & B & OR & $95 \% \mathrm{CI}$ & $p$ value \\
\hline WBC count $\left(/ \mathrm{mm}^{3}\right) \geq 9,900.0$ & 2.274 & 9.722 & $2.566,36.836$ & 0.001 & 1.763 & 5.828 & $1.378,24.640$ & 0.017 \\
\hline Eosinophil count $\left(/ \mathrm{mm}^{3}\right) \geq 2,400.0$ & 2.091 & 8.095 & $2.209,29.660$ & 0.002 & 1.515 & 4.552 & $1.086,19.077$ & 0.038 \\
\hline Haemoglobin (g/dL) & -0.137 & 0.872 & $0.602,1.264$ & 0.470 & & & & \\
\hline Platelet count $\left(\times 1,000 / \mathrm{mm}^{3}\right)$ & 0.002 & 1.002 & $0.997,1.008$ & 0.405 & & & & \\
\hline Blood urea nitrogen (mg/dL) & 0.007 & 1.007 & $0.929,1.091$ & 0.872 & & & & \\
\hline Creatinine $(\mathrm{mg} / \mathrm{dL})$ & -0.162 & 0.851 & $0.597,1.213$ & 0.372 & & & & \\
\hline Alkaline phosphatase (IU/L) & -0.004 & 0.996 & $0.983,1.010$ & 0.601 & & & & \\
\hline $\operatorname{ESR}(\mathrm{mm} / \mathrm{hr})$ & -0.002 & 0.998 & $0.978,1.018$ & 0.841 & & & & \\
\hline CRP (mg/L) & 0.004 & 1.004 & $0.984,1.024$ & 0.679 & & & & \\
\hline
\end{tabular}

HES: hypereosinophilic syndrome; WBC: white blood cell; ESR: erythrocyte sedimentation rate; CRP: C-reactive protein.

A

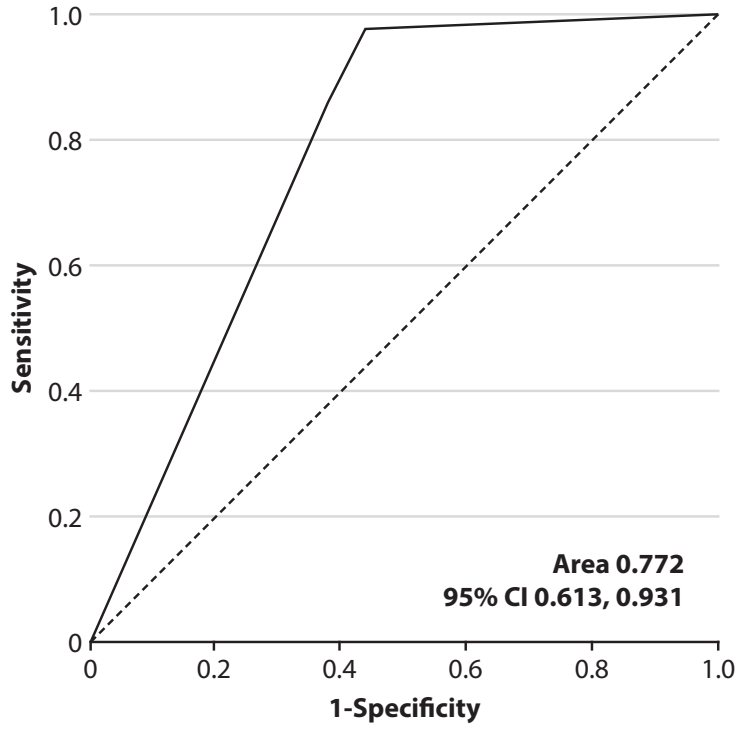

Figure 1. Relative risk for HES based on HSLI

A) The optimal cut-off of HSLI for HES was set at 4.25 (Area $0.772,95 \%$ CI $0.613,0.931$ ).

B) HES appeared more frequently in patients with HSLI at diagnosis $\geq 4.25$ than those without. In addition, patients with HSLI at diagnosis $\geq 4.25$ exhibited a significantly high RR for HES at diagnosis, compared to those without (RR 51.429).

HES: hypereosinophilic syndrome; HSLI: HES-suggesting laboratory index; RR: relative risk.

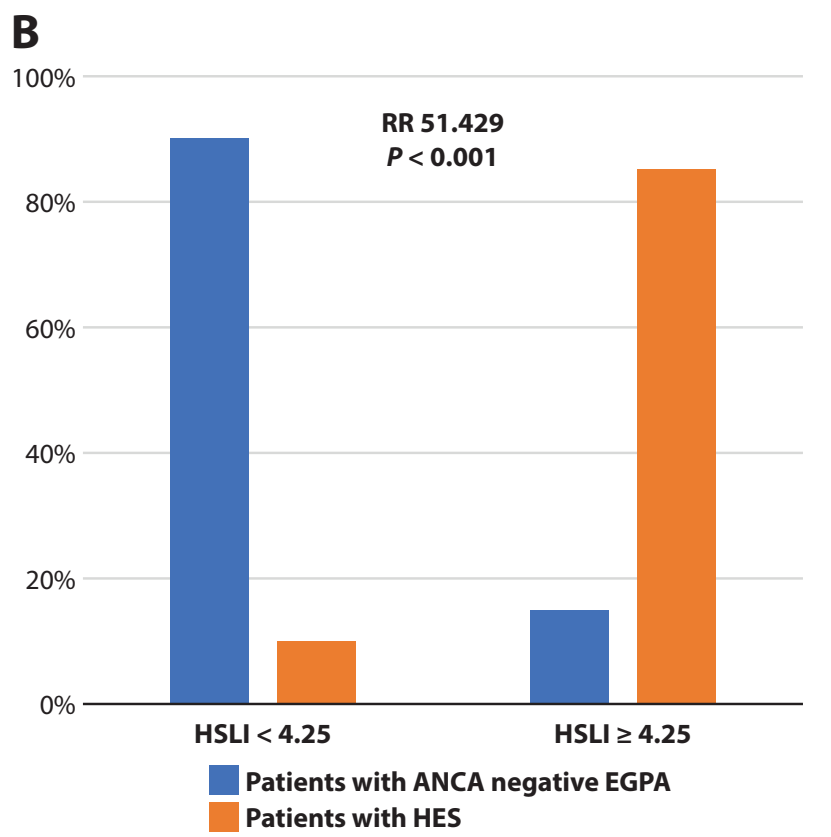


of HSLI for HES, 47 patients belonged to the group of HSLI $\geq 4.25$. HES appeared more frequently in patients with HSLI at diagnosis $\geq 4.25$ than those without $(85.1 \%$ vs. $10.0 \%, P<$ 0.001). In addition, patients with HSLI at diagnosis $\geq 4.25$ exhibited a significantly high RR for HES at diagnosis, compared to those without (RR 51.429, 95\% CI 5.605, 471.890) (Figure 1B).

\section{Discussion}

In this study, we demonstrated the predictive potential of WBC and eosinophil counts at diagnosis for HES. Furthermore, we developed an equation of HSLI to identify HES using the cut-offs of WBC and eosinophil counts at diagnosis for the presence of HES. Compared to each WBC count and eosinophil count at diagnosis as an independent predictor of HES in the multivariable analysis, HSLI consists of two predictors such as WBC and eosinophil counts, thus its accuracy and reliability might be higher than those of each independent predictor. We conducted the ROC curve using HSLI $\geq 4.25$, WBC count at diagnosis $\geq 9,900.0 / \mathrm{mm}^{3}$ and eosinophil count at diagnosis $\geq 2,400.0 / \mathrm{mm}^{3}$ at for HES and compared the area under the curve (AUC) among them. AUC of HSLI $\geq 4.25$ (area $0.739,95 \%$ CI $0.583,0.896$ ) was significantly larger than those of WBC at diagnosis $\geq 9,900.0 / \mathrm{mm}^{3}$ (area $0.727,95 \%$ CI $0.570,0.884$ ) and eosinophil count at diagnosis $\geq 2,400.0 / \mathrm{mm}^{3}$ (area 0.769, 95\% CI 0.609, 0929) (Supplementary Figure 1). With this result, we conclude that a new index of HSLI is more reliable to predict HES than either WBC or eosinophil count at diagnosis.

How could WBC count and eosinophil count at diagnosis be clues to predict HES compared to ANCA-negative EGPA? Unlike HES, EGPA has three phases as the following: i) the first phase is an allergic stage, which is characterised by asthma and sinusitis; ii) the second phase is an eosinophilic stage of which features are peripheral hypereosinophilia and occasional eosinophilic infiltration to lungs and gastrointestinal tracts; and iii) the third phase is a vasculitic stage where necrotising inflammation of small vessels in various organs, leading to major organ damages. ${ }^{18,19}$ Asthma, sinusitis and peripheral blood eosinophilia are mainly observed in allergic or eosinophilic phases, whereas unfixed pulmonary infiltration and peripheral neuropathies often occurs in vasculitic phase. Histology confirmation is also mainly performed in vasculitic phase at which major organ involvement is more apparent than other phases. For these reasons, most EGPA patients are classified as EGPA in the vasculitic phase and exhibited the lower eosinophil along with WBC counts at diagnosis than HES patients. In addition, In the late phase, $\mathrm{T}_{\mathrm{H}} 1$ and $\mathrm{T}_{\mathrm{H}} 17$ mediated granulomatous necrotising vasculitis exceeds $\mathrm{T}_{\mathrm{H}} 2$ mediated inflammation, which may also contribute the reduced eosinophil count at diagnosis in EGPA patients compared to HES patients. ${ }^{4,20}$

Of 41 HES patients, 10 patients (24.4\%) had asthma at the time of diagnosis. Based on the definition of HES, medical history of allergic diseases should be excluded, whereas the 1990 ACR criteria for EGPA include an item of asthma or a history of asthma. ${ }^{4,5}$ If so, should these HES patients with asthma be reclassified as EGPA? A previous study reported that asthma developed prior to HES occurrence in $25 \%$ of HES patients. ${ }^{21}$ In this study, asthma was observed in $24.4 \%$ of HES patients at diagnosis. Moreover, asthma could be found as an end-organ manifestation of lungs along with infiltrative eosinophilic diseases in HES patients. ${ }^{1,8}$ Therefore, although ANCA-negative EGPA patients exhibited asthma much more frequently than HES patients in the comparison analysis in this study, no one can tell whether the current asthma is a cause of EGPA or a consequence of HES. For this reason, asthma was not included in the multivariable logistic regression analysis with WBC and eosinophil counts at diagnosis.

In terms of items of the 1990 ACR criteria for EGPA other than asthma, both frequencies of paranasal sinusitis and peripheral neuropathy in ANCA-negative EGPA patients were

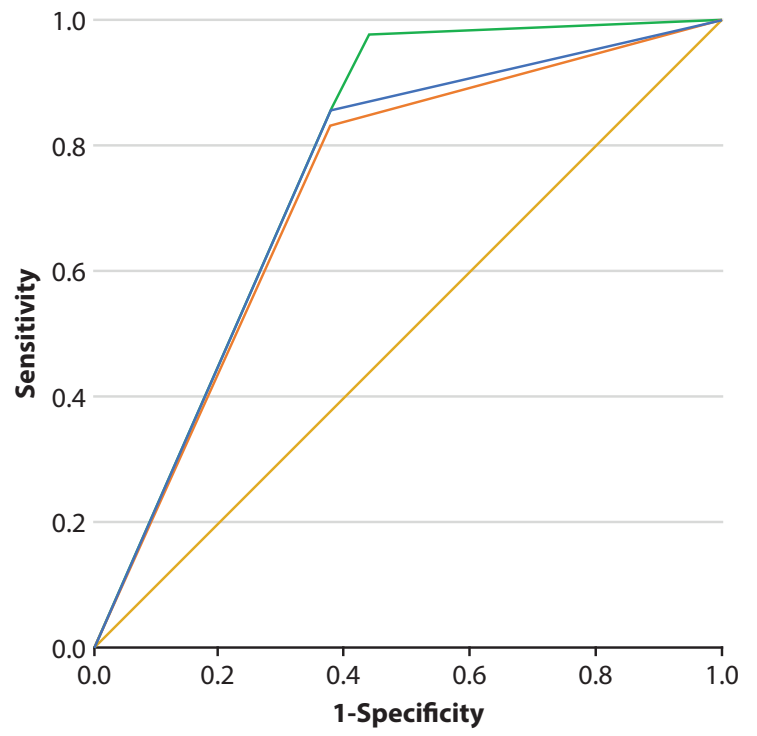

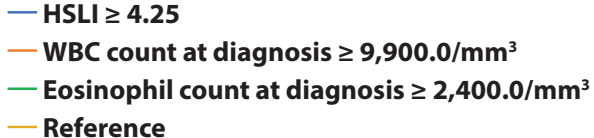

- Reference

\begin{tabular}{|ccc}
\multicolumn{1}{c}{ Variables } & Area & $\mathbf{9 5 \%}$ CI \\
\hline HSLI at diagnosis $\geq 4.25$ & 0.739 & $0.583,0.896$ \\
\hline WBC count at diagnosis $\geq 9,900.0 / \mathrm{mm}^{3}$ & 0.727 & $0.570,0.884$ \\
\hline Eosinophil count at diagnosis $\geq 2,400.0 / \mathrm{mm}^{3}$ & 0.769 & $0.609,0.929$ \\
\hline
\end{tabular}

Supplementary Figure 1. Comparison of the area under the receiver operator characteristic (ROC) curve among the cut-offs of WBC and eosinophil counts and HSLI for HES.

WBC: white blood cell; HSLI: HES-suggesting laboratory index; HES: hypereosinophilic syndrome. 
significantly higher than those in HES patients (Table 1). However, these two items do not provide a definitive clue to the differential diagnosis of the two diseases if they are understood in the same context as asthma. Paranasal sinusitis in HES patients has occasionally been reported and it was observed in $31 \%$ of HES patients in this study. ${ }^{22}$ Moreover, since the peripheral nervous system is belonging to major damaged end organs in HES, peripheral neuropathy is likely to occur in HES patients. ${ }^{1,4}$ However, eosinophilic infiltration was not histologically confirmed in any HES patients. This result might be attributed to the definition of HES, in which the first requirement is peripheral blood eosinophil $\geq 1,500 / \mathrm{mm}^{3}$ or end-organ damage regardless of blood eosinophil count. ${ }^{4}$ In this study, all HES patients had blood eosinophil count of $1,500 / \mathrm{mm}^{3}$ or greater and none of them underwent tissue biopsy. Therefore, we did not include paranasal sinusitis, peripheral neuropathy and extravascular eosinophils in the multivariable logistic regression analysis with WBC and eosinophil counts at diagnosis.

In terms of organ-specific clinical manifestations, except ENT and nervous manifestations, renal manifestation was rarely observed in any HES patients compared to ANCA-negative EGPA. ${ }^{10}$ A previous study reported the frequency of renal involvement of HES as $0 \%$ and another study described renal symptoms in HES patients as a result of thromboembolism. ${ }^{23,24}$ Therefore, we included renal manifestation in the multivariable logistic regression analysis with WBC and eosinophil counts at diagnosis. However, renal manifestation did not alter the statistical significance of the association of WBC count at diagnosis (OR 8.654, 95\% CI 1.797, 41.664) and eosinophil count at diagnosis (OR 6.884, 95\% CI 1.431, 33.111) with the presence of HES.

As we mentioned, EGPA is included in the category of associated HES.,12 Nevertheless, there are two medical reasons for applying HSLI for patients suspected of both HES and ANCA-negative EGPA. The first reason is to select induction therapeutic regimens for HES and ANCA-negative EGPA. The selection of induction therapeutic regimens is based on the category in HES patients, whereas it is based on FFS (2009) in EGPA patients. ${ }^{10,12-14}$ For instance, imatinib mesylate should be considered in patients with platelet-derived growth factor receptor- $\alpha$ (PDGFRA)-positive myeloproliferative variant HES, whereas, cyclophosphamide might be considered in EGPA patients with FFS $\geq 1$. $^{1,10,12}$ The second reason is to find EGPA patients with a more allergic component. Recently, humanised anti-IL-5, mepolizumab, has been developed and approved by the Food and Drug Administration of the United States in EGPA patients. ${ }^{25}$ In a clinical trial, mepolizumab increased the proportion of participants in remission and reduced glucocorticoid use. ${ }^{26}$ Mepolizumab induces the arrest of eosinophil maturation in the bone marrow and reduces eosinophil progenitors and maturation in the peripheral blood, resulting in a decrease in eosinophil count. ${ }^{27}$ For this reason, mepolizumab may theoretically be more effective in eosinophilic phase of EGPA than vasculitic phase of EGPA. In this study, there was a difference in the median eosinophil count between ANCA-negative EGPA patient with and without HSLI $\geq 4.25\left(13,120.0 / \mathrm{mm}^{3}\right.$ vs. $1,240 / \mathrm{mm}^{3}, P=$ 0.045). Therefore, we expect that the cut-off of HSLI for HES might be useful for finding proper ANCA-negative EGPA patients in whom mepolizumab is effective.

In this study, we first proposed an index of HSLI for distinguishing between HES and ANCA-negative EGPA and demonstrated the predictive potential of HSLI $\geq 4.25$ for HES. The cut-off of HSLI for HES may be changeable according to ethnicity and populations and so it is not appropriate to apply HSLI $\geq 4.25$ to all patients suspected of both HES and ANCA-negative EGPA. Instead, this study suggested a method to obtain the equation of the cut-off of HSLI for HES and thus the cut-offs based on ethnicity and populations can be used in all patients suspected of both HES and EGPA.

Our study has several limitations. First of all, the number of this study was not large enough to generalise these findings to all EGPA patients. For this reason and given the ethnic difference, the relevance of the HSLI should be validated in larger population samples from other ethnicities. However, the results of this study were not validated by other ethnic populations beyond the Korean population..$^{28}$ Moreover, it is unclear whether HSLI may be identically applied in eosinophilia subjects without or only with mild systemic symptoms and will be positive throughout the clinical course prior to treatment; its reproducibility could be also variable according to the timepoint when the assessment was made. Also, given that is a retrospective study, we could not control or minimise the confounding factors which were not written in the medical records. Furthermore, at the time of diagnosis, we did not perform bone marrow biopsy in all patients, although the bone marrow histology and genetic analysis would help to discern HES from EGPA. Additionally, it is controversial whether white blood cell and absolute eosinophil counts is an indicator of active inflammation or merely suggests organ injury in these study population. Last, although asthma may be a sine qua non for EGPA, we quoted studies with the high incidence of asthma in HES and this may lead to weaknesses in study design. Nevertheless, we believe that our study has clinical meanings in that we suggested a method to obtain the equation of the cut-off of HSLI for HES. Future prospective studies with a larger number of HES patients and ANCA-negative EGPA patients will provide more reliable information on the clinical usefulness of a newly developed HSLI in distinguishing between HES and ANCA -negative EGPA.

In conclusion, we provided a new index for distinguishing between HES and EGPA and suggested that the cut-off of HSLI derived from WBC and eosinophil counts at diagnosis is an independent predictor of HES in patients suspected of both HES and ANCA-negative EGPA.

\section{Acknowledgments}

None

\section{Conflicts of interest}

The authors declare they have no conflicts of interest. 


\section{Author contributions}

SSA designed the report and wrote the paper; JY participated in data acquisition and interpretation; YBP and JWP drafted and revised the manuscript; JHL and SWL designed the concept and approved the final paper. All authors have taken care to ensure the integrity of this work, and the final manuscript has been seen and approved by all authors.

\section{Funding}

This research was supported by a grant from the Korea Health Technology R\&D Project through the Korea Health Industry Development Institute, funded by the Ministry of Health and Welfare, Republic of Korea (HI14C1324).

\section{References}

1. Curtis C, Ogbogu P. Hypereosinophilic Syndrome. Clin Rev Allergy Immunol. 2016;50:240-51.

2. Chusid MJ, Dale DC, West BC, Wolff SM. The hypereosinophilic syndrome: analysis of fourteen cases with review of the literature. Medicine (Baltimore). 1975;54:1-27

3. Klion AD, Bochner BS, Gleich GJ, Nutman TB, Rothenberg ME, Simon $\mathrm{HU}$, et al. Approaches to the treatment of hypereosinophilic syndromes: a workshop summary report. J Allergy Clin Immunol. 2006;117:1292-302.

4. Simon HU, Rothenberg ME, Bochner BS, Weller PF, Wardlaw AJ, Wechsler ME, et al. Refining the definition of hypereosinophilic syndrome. J Allergy Clin Immunol. 2010;126:45-9.

5. Churg J, Strauss L. Allergic granulomatosis, allergic angiitis, and periarteritis nodosa. Am J Pathol. 1951;27:277-301.

6. Jennette JC, Falk RJ, Bacon PA, Basu N, Cid MC, Ferrario F, et al. 2012 revised International Chapel Hill Consensus Conference Nomenclature of Vasculitides. Arthritis Rheum. 2013;65:1-11.

7. Masi AT, Hunder GG, Lie JT, Michel BA, Bloch DA, Arend WP, et al. The American College of Rheumatology 1990 criteria for the classification of Churg-Strauss syndrome (allergic granulomatosis and angiitis). Arthritis Rheum. 1990;33:1094-100.

8. Dulohery MM, Patel RR, Schneider F, Ryu JH. Lung involvement in hypereosinophilic syndromes. Respir Med. 2011;105:114-21.

9. Noh HR, Magpantay GG. Hypereosinophilic syndrome. Allergy Asthma Proc. 2017;38:78-81.

10. Millet A, Pederzoli-Ribeil M, Guillevin L, Witko-Sarsat V, Mouthon L. Antineutrophil cytoplasmic antibody-associated vasculitides: is it time to split up the group? Ann Rheum Dis. 2013;72:1273-9.

11. Furuta S, Iwamoto T, Nakajima H. Update on eosinophilic granulomatosis with polyangiitis. Allergol Int. 2019;68:430-6.

12. Cogan E, Roufosse F. Clinical management of the hypereosinophilic syndromes. Expert Rev Hematol. 2012;5:275-89; quiz 90.
13. Hsieh FH. Hypereosinophilic syndrome. Ann Allergy Asthma Immunol. 2014;112:484-8.

14. Guillevin L, Pagnoux C, Seror R, Mahr A, Mouthon L, Le Toumelin P. The Five-Factor Score revisited: assessment of prognoses of systemic necrotizing vasculitides based on the French Vasculitis Study Group (FVSG) cohort. Medicine (Baltimore). 2011;90:19-27.

15. Khoury P, Zagallo P, Talar-Williams C, Santos CS, Dinerman E, Holland NC, et al. Serum biomarkers are similar in Churg-Strauss syndrome and hypereosinophilic syndrome. Allergy. 2012;67:1149-56.

16. Khoury P, Makiya M, Klion AD. Clinical and Biological Markers in Hypereosinophilic Syndromes. Front Med (Lausanne). 2017;4:240.

17. Watts R, Lane S, Hanslik T, Hauser T, Hellmich B, Koldingsnes W, et al. Development and validation of a consensus methodology for the classification of the ANCA-associated vasculitides and polyarteritis nodosa for epidemiological studies. Ann Rheum Dis. 2007;66:222-7.

18. Lanham JG, Elkon KB, Pusey CD, Hughes GR. Systemic vasculitis with asthma and eosinophilia: a clinical approach to the Churg-Strauss syndrome. Medicine (Baltimore). 1984;63:65-81.

19. Vaglio A, Casazza I, Grasselli C, Corradi D, Sinico RA, Buzio C. Churg-Strauss syndrome. Kidney Int. 2009;76:1006-11.

20. Tsurikisawa N, Saito H, Tsuburai T, Oshikata C, Ono E, Mitomi H, et al. Differences in regulatory $\mathrm{T}$ cells between Churg-Strauss syndrome and chronic eosinophilic pneumonia with asthma. J Allergy Clin Immunol. 2008;122:610-6.

21. Lefevre G, Ackermann F, Kahn JE. Hypereosinophilia with asthma and systemic (non-vasculitic) manifestations: Eosinophilic granulomatosis with polyangiitis or hypereosinophilic syndrome? Autoimmun Rev. 2017; 16:208-9.

22. Kobayashi M, Kubota T, Uemura Y, Taguchi H. A case of hypereosinophilic syndrome presenting with chronic cough successfully treated with imatinib. Respirology. 2009;14:302-4.

23. Liapis H, Ho AK, Brown D, Mindel G, Gleich G. Thrombotic microangiopathy associated with the hypereosinophilic syndrome. Kidney Int. 2005;67:1806-11.

24. Fauci AS, Harley JB, Roberts WC, Ferrans VJ, Gralnick HR, Bjornson $\mathrm{BH}$. NIH conference. The idiopathic hypereosinophilic syndrome. Clinical, pathophysiologic, and therapeutic considerations. Ann Intern Med. 1982;97:78-92.

25. Dispenza MC, Bochner BS. Diagnosis and Novel Approaches to the Treatment of Hypereosinophilic Syndromes. Curr Hematol Malig Rep. 2018;13:191-201.

26. Wechsler ME, Akuthota P, Jayne D, Khoury P, Klion A, Langford CA, et al. Mepolizumab or Placebo for Eosinophilic Granulomatosis with Polyangiitis. N Engl J Med. 2017;376:1921-32.

27. Fu Z, Yu C, Wang L, Gao K, Xu G, Wang W, et al. Development of a robust reporter gene based assay for the bioactivity determination of IL-5-targeted therapeutic antibodies. J Pharm Biomed Anal. 2018;148:280-7.

28. Shimoi T, Shojima K, Murota A, Takizawa Y, Maruyama J, Setoguchi K. Clinical and pathological features of Churg Strauss syndrome among a Japanese population: a case series of 18 patients. Asian Pac J Allergy Immunol. 2012;30:61-70. 